\title{
STUDYING COMMUNICATIONS RESILIENCY IN EMERGENCY PLANS
}

\author{
Cristina Ruiz-Martin \\ Gabriel Wainer \\ Dept. of Systems and Computer Engineering \\ Carleton University \\ Ottawa, Canada \\ \{cristinaruizmartin, gwainer\}@sce.carleton.ca
}

\author{
Adolfo Lopez-Paredes \\ INSISOC \\ Universidad de Valladolid \\ Valladolid, Spain \\ aparedes@eii.uva.es
}

\begin{abstract}
Recent disasters have shown that hazards can be unpredictable and can have catastrophic consequences. Emergency plans are key to dealing with these situations and communications play a key role in emergency management. In this paper, we provide a formalism to design resilient emergency plans in terms of communications. We exemplify how to use the formalism using a case study of a Nuclear Emergency Plan.
\end{abstract}

Keywords: resilience, DEVS, communications, ABM, network theory

\section{INTRODUCTION}

Recent disasters, such as the Earthquake and Tsunami in Japan in 2011 (MacKenzie, Santos, and Barker, 2012), the Darfield Earthquake in New Zealand in 2010 (Whitman, et. al, 2014; Kachali et. al 2012), or the accident at TEPCO's Fukushima Nuclear Power Plant (NPP) in 2011 (Omoto, 2013; Langlois, 2013) showed unpredictability. To overcome these situations, governments and companies design Emergency Plans. However, it is not straightforward how to evaluate in advance if the plan is going to work in case of an emergency. The analysis of such disasters provided insights into the critical elements during the emergency, and many authors highlighted the importance of communications in order to manage an emergency. For example, after the Darfield earthquake in September 2010 (Whitman et. al 2014), it was found that relationships within the staff were among the critical factors. After Hurricane Katrina in 2005, the organizations that used Information and Communication Technologies to recover exhibited a higher level of resilience (Chewning, Lai, and Doerfel, 2012). Here, we discuss the application of a method to design resilient communications mechanisms for emergency plans, based on (Ruiz-Martin, Wainer, and Lopez-Paredes, 2018a). The method, based on diffusion processes in multiplex networks, is used to study the resilience of communications in a nuclear emergency plan.

In section 2 we explain the related work on how to design resilient emergency plans with a focus on the communications. In section 3, we explain the method we propose to design resilient emergency plans in terms of communications. In section 4, we apply the method proposed in section 3 to the case study of a nuclear emergency plan. Finally, in section 5, we present the conclusions of this work.

\section{RELATED WORK}

Governments are investing in resilient institutions, communities, organizations, and individuals; the European Commission (2017) has identified resilience among the top five EU's priorities; the Government of Canada (2014) is concerned about climate resilience and its impact.

We understand as resilience as a measurable combination of characteristics, abilities, capacities or capabilities that allows an organization to withstand known and unknown disturbances and still survive 
(Ruiz-Martin, López-Paredes, and Wainer, 2018b). In the context of the communications for emergency plans, it means, that the devices should allow the individuals involved in the emergency to receive the information needed to perform their assigned tasks in order to solve the emergency.

After the accident at TEPCO's Fukushima NPP, several problems in the emergency plan and crisis management were identified: loss of functionality at the off-site emergency management center, no availability of the communication systems; insufficient warning to the population, evacuation, poor communication and information management (Omoto, 2013). The International Atomic Energy Agency suggested the need for improvements, including strengthening response arrangements, transparency, and, in the context of this research, the effectiveness of the communications mechanisms (Langlois, 2013). Following the Darfield earthquake, (Whitman et. al, 2014) presented a survey discussing helpful factors in mitigating the disruption in the operations after the earthquake: well-designed and well-built buildings, the relationship with staff and the capability to restore critical services quickly or not to get them interrupted. After Hurricane Katrina, industries in the area (fishing, cotton, rice, and sugar cane) were destroyed. In (Chewning, Lai, and Doerfel, 2012), the authors studied how organizations with the ability to use Information and Communication Technology in their recovery were more resilient. Likewise, after the World Trade Center attacks, New York City departments lost their primary emergency operation center. In (Kendra and, Wachtendorf, 2003), the authors also pointed out the importance of communication systems.

Resilience is also improved by better decision support systems. For example (Espinosa-Paredes et. al, 2008) present a model to study abnormal situations in nuclear power plants using Fuzzy Cognitive Maps (FCM). In (De Maio et. al, 2011), the authors also use FCM to build a decision support system, including knowledge-based systems with information from software, web technologies and social aspects. (Mendonça et. al, 2006) presents a gaming simulation model to get data from emergency response scenarios while training the first responders in virtual environments.

In (Ruiz-Martin et. al, 2016; Bouanan 2016) we combined Discrete Event System Specification (DEVS) (Zeigler et al. 2000), Agent-Based Modeling (ABM) and Network Theory to study information diffusion processes in multiplex networks using a server-proxy architecture where the servers represent the behavior of the nodes, and the proxies define how information spreads among the different communication links. The main drawback is that the representation of complex behavioral rules for individuals is complex. Additionally, the Server-Proxy architecture does not allow to model all the properties of all diffusion models. For example, the individuals in an organization could use different devices (and their networks).

Instead, in (Ruiz-Martin et al. 2018a) we introduced an Architecture for Diffusion Processes in Multiplex dynamic networks, which combines Network Theory, to define the network that supports the diffusion process, with Agent-Based Modeling (ABM) to define the behavior of the entities involved, and a formal specification for simulation modeling using DEVS. In the following sections we show how to use these tools to study and improve the resilience of communications in emergency plans.

\section{A METHOD TO DESIGN RESILIENT COMMUNICATIONS IN EMERGENCY PLANS}

Our architecture, presented in Figure 1 and detailed in (Ruiz-Martin et al. 2018a), includes six components:

- Diffusion experiment data collection: this step formalizes the process by which a modeler obtains all the requirements, specifications, and data available for the communication system under study.

- Network model: an organized representation of the Diffusion experiment data using Network Theory (Note: this is NOT a Computer Network; it is a model that provides a formal representation of the relations between the communication components of the system).

- Agent-Based model: it represents the behavior of those starting the diffusion process, the objects they use for diffusing the element, and the properties of the relationships among these objects.

- Diffusion Abstract Model (DAM): an abstract and formal representation of the Diffusion experiment data that matches elements in both the Network and the Agent-based models. 
- Diffusion Computerized Model (DCM): a software implementation of the DAM used to run simulations.

- Results Analysis using different methods and data visualization tools

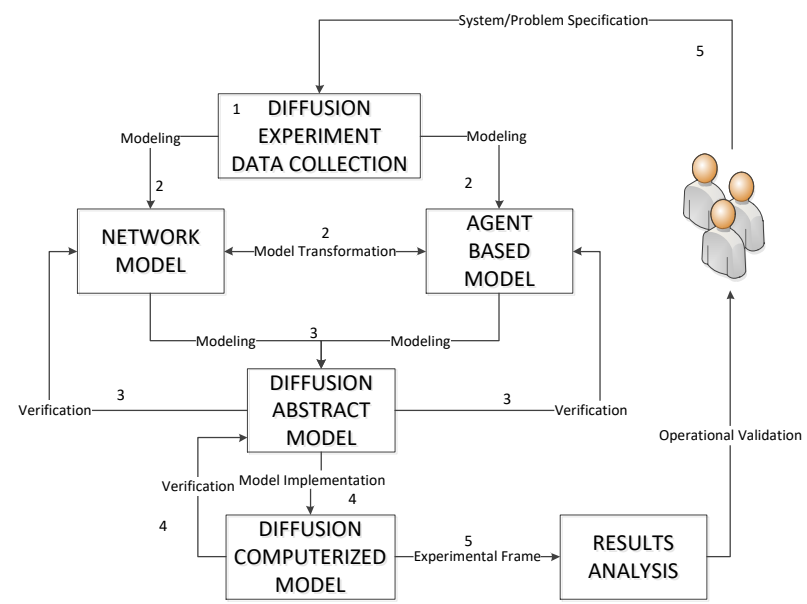

Figure 1: Architecture to simulate diffusion processes in multiplex networks.

As we mentioned, we can model the structure of the emergency plan and the communications among its participants as an information diffusion process over a network. In the rest of the section, we explain how to adapt each component of the architecture to the specific case of communications in emergency plans.

\subsection{Diffusion experiment data collection}

We need to include all the requirements, specifications, and data available for the communications in the emergency plan. The minimum elements include:

- The set of possible messages to be transmitted during the emergency. These are the elements to be diffused (commands, acknowledgments, information, etc.).

- The users involved in managing the emergency and their behavior. Normally, the end users are individuals, but we may also have Artificial Intelligent (AI) Systems that generate messages.

- How and/or who generates the set of possible messages to be transmitted over time.

- How the messages transmitted affect the behavior of those involved in the emergency.

- The mechanisms to transmit the messages, including characteristics and connections. For example, mobile devices, internet, landline, etc.

- The diffusion mechanisms that can be used by the users.

- The variables to study. For instance, for the resilience of the communications, we want to identify how many end users are isolated, where are the bottlenecks, messages lost, etc.

- The scenarios to be analyzed. We should study the ideal scenarios when all the communication mechanisms work as expected, and others where we introduce several types of failures.

\subsection{Network Model}

The nodes of the Network Model represent the users of the emergency system. The links between nodes represent if there is any kind of communication mechanism available between two of the nodes. The type of link represent which specific communication mechanism is available between the two nodes.

\subsection{AGENT BASE MODEL (ABM)}

The simplest definition for agent behavior is shown in figure 2, where we show a part of user's behavior implemented in XML. The agent behavior is defined between the tags $<$ AgentBehavior $>$, with attributes:

- User Id.

- MyDevices: the different devices available to the user to communicate during the emergency. 
- MyRelations: the agent's output connections (the users that can be reached by this specific agent).

- BehaviorRules: rules of the communication process (the rules defined in the emergency plan for communications and expected behavior of the agents; each Rule element can have different parameters).

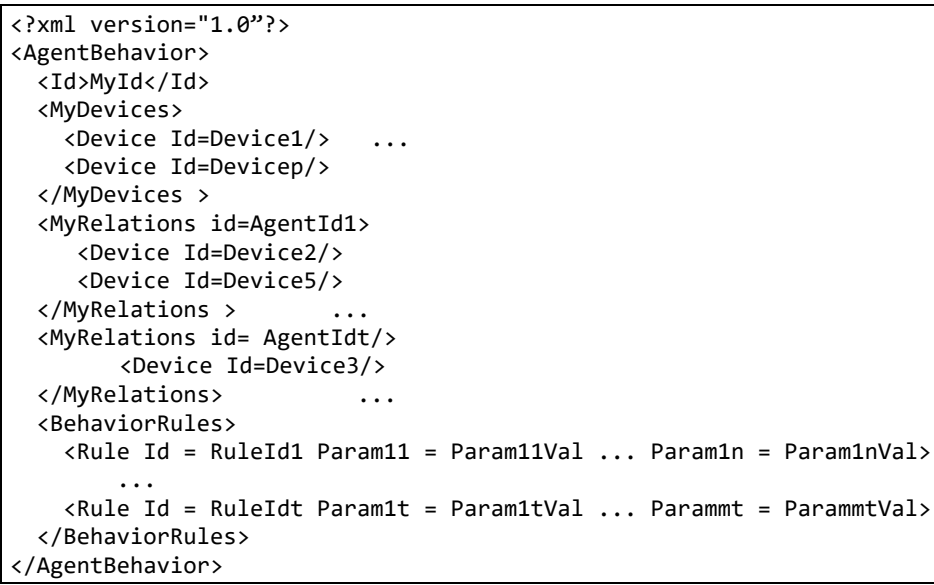

Figure 2: Most simple ABM behavior definition.

\subsection{DIFFUSION ABSTRACT MODEL (DAM)}

The DAM to study the communications in emergency plans (figure 3) is an instantiation of a generic DAM that defines diffusion processes in multiplex dynamic networks (Ruiz-Martin et al. 2018a). Here, we instantiate the components of the DAM as DEVS atomic or coupled models. Each component is defined using the information in the network model and the XML agent implementation explained earlier.

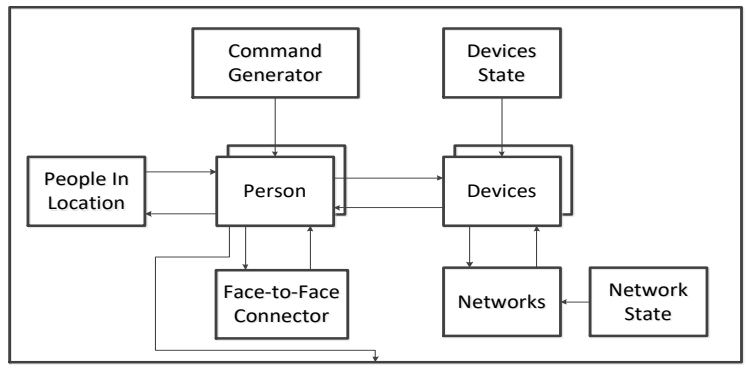

Figure 3: DAM for the study of communications in emergency plans.

Here, Person represents the people or AI systems involved in the emergency plan. Devices represents the communication mechanism (i.e. phones, computers, etc.) people use to communicate with each other. Communication Networks represents the networks that connect the different devices (for instance, the Internet, the telephone network, etc.). Face-to-Face Connector enables direct communications between people in the same location. People In Location model updates the list of people that are in a specific location. Device State and Network State respectively define if a device or a network is broken and for how long. Finally, Command Generator generates the messages to be sent in case of an emergency.

In the rest of the section, we detail how the Person model is defined as it is the most complex one. It is a Coupled model presented in figure 4 that includes 12 components. The main core of the Person model is Behavior Rules. It is defined as a generic coupled model using the agent behavior defined in the XML file. It models how the person makes decisions about the messages and how it executes tasks based on the behavior defined in the XML file, the devices that are activated, the messages received, the users around, and a to-do list. The filters are atomic models that select which of the messages are targeted to each Person based on the Id of the agent defined in the XML file (as messages are broadcasted, they have to decide if they are the destination). In-Situ Switch is an atomic model that classifies the incoming message based on the state of the Switch. The Switch can be in three states: decide, send and receive. Base on the switch state, it redirects the messages received from the In-Situ input port to the appropriate out port: Send Out that is 
connected to Sending Behavior using In-situ, Receive Out that is connected to Receiving Behavior Using In-situ or, Decide Out that is connected to Behavior Rules.

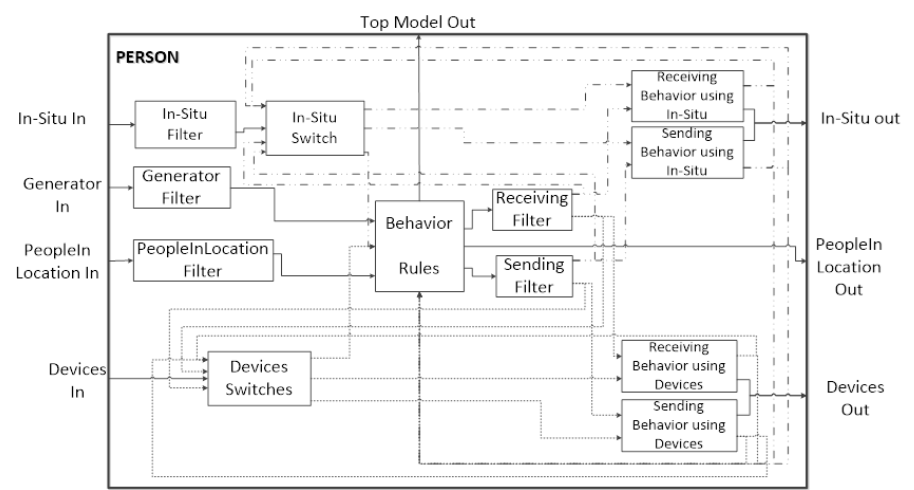

Figure 4: Coupled model definition of the Person component.

Devices Switches is a coupled model instantiated based on the different types of devices that a person can use. Those devices are defined in the XML agent implementation, therefore, Devices Switches in different Persons may have different number of components. Their behavior is similar to In-Situ Switch. Receiving/ Sending Filters classify the instructions of Behavior Rules based on what communications are used (i.e. InSitu or Device communications). They resend these instructions through the appropriate output port. Receiving Behavior using In-Situ represents the process a person follows to receive a message/communication. Similarly, Sending Behavior using In-Situ represents the process a person follows to communicate a message. The models Sending/Receiving Behavior using Devices are coupled models that represent the process a person follows to receive/send a message/communication using specific devices. They are generic models that are instantiated for each Person model using two attributes of the agent behavior defined in the XML: MyDevices and Id. We decide which atomic models we include (i.e. Call using Mobile, Send Fax, etc.) based on MyDevices attribute. These atomic models are instantiated with the $I d$ attribute of the Person to identify the person that is calling, sending a fax, etc.

In figure 5, we show an example of the Sending Behavior using Devices coupled model instantiated for a person with a fax and a mobile. The Switches Filter, Sending Filter and Sending Sink are common for all instantiations. Switches Filter and Sending Filter redirect the messages to the proper components. Sending Sink collects all the messages that a person tries to send using a device that she does not possess. The models that change from one instantiation to others are the ones representing the use of different devices.

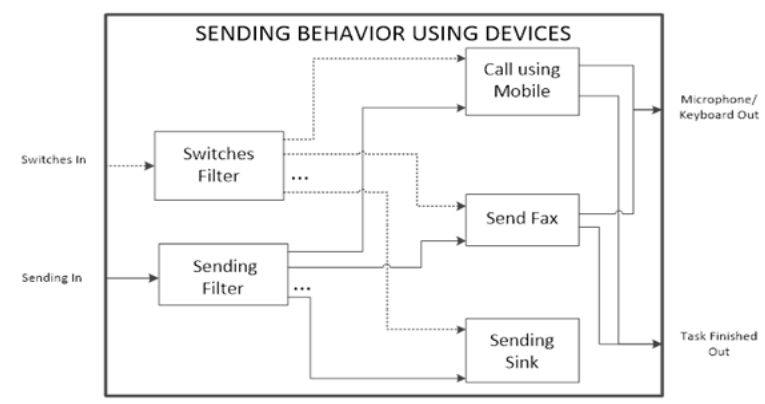

Figure 5: Sending Behavior using Devices coupled model.

\subsection{DIFFUSION COMPUTERIZED MODEL (DCM)}

We built the DCM for studying the resilience of communications using Cadmium (Vicino et. al, 2019). We defined a parser to automatically implement the DCM. The program uses all the atomic and coupled models previously defined and the information in the agent XML definition. The output of the parser is the DCM written in $\mathrm{C}++$ and ready to be executed in Cadmium. An advantage of this approach is that we update the 
behavior of the agents directly in the XML to define new scenarios. Our parser automatically generates a new DCM. However, if we change the structure of the agents in the XML, we need to define a new parser.

\section{COMMUNICATIONS RESILIENCE FOR A NUCLEAR EMERGENCY PLAN}

This section presents a case study applying the concepts above to study the resiliency of the communications of an Emergency Plan defined for a Nuclear Power Plant (NPP) in Spain (the location of the NPP and the plan are confidential; we discuss aspects that can be shared with the public). First, we collected the Nuclear Emergency Plan (NEP) information from the Civil Protection Agency. The plan is designed to define the actions and the communication mechanisms in case of an accident in the NPP. The comprehensive explanation of the Requirements Document for the NEP organization can be found in (Ruiz-Martin, 2013).

The NEP is a complex organizational structure that involves many individuals. At the core, we have the NEP Director, who takes the decisions to manage the emergency. However, as the emergency evolves, higher National Government ranks, such as the President, can take the position of the NEP Director. The NSC President (Nuclear Safety Commission), and the PENCRA Director (Central Response and Support Nuclear Emergency Plan) are at the same level as the NEP Director. The NSC Inspector at the NPP is in direct communication with the NEP Director. An Advisory Committee must advise the NEP Director. The Information and Communication Cabinet is in charge of communications with the media. The Executive Body is composed of the leaders of the Radiological, Health, Logistical Support, Public Security and Order, and Technical Assistance and Coordination groups. The Executive Body receives commands of the NEP director and executes them. Each group from the Executive Body has a predefined structure and function. For example, the Radiological group is in charge of radiological control of the population and first responders. The Logistical Support Group provides food, evacuation, and coordination. The Public Security and Order Group controls the access to the emergency area. The Technical Assistance and Coordination group manage evacuation or confinement in different municipalities. In each group, there are heads at various levels, first responders and backup teams.

The communication systems in the NEP include landline and mobile phones, fax, mixed radio/phone networks, satellite, Internet, Remer and Reman radio channels, and in-situ communication. Emergency managers and first responders can also use a military communication system. However, this is not available until the NEP Director makes a request and the entire infrastructure is deployed (we do not consider it in our model; this is a backup plan in case everything else fails, and it has not been studied). The NEP defines every possible command (more than 30 ) to be handled in the case of an emergency. The NEP director selects what to do based on the evolution of the emergency.

\subsection{Network Model}

After the Requirements Document was completed, we used it to define the Network Model. To build the network, we use the following information from the Requirements Document: the users involved in the NEP, and the systems they can use to communicate with each other.

Table 1: Nodes Ids and labels.

\begin{tabular}{|ll|}
\hline Id & Label \\
\hline $\mathbf{1}$ & NEP Director \\
\hline $\mathbf{2}$ & PENCRA Director \\
\hline $\mathbf{3}$ & NSC Inspector at the NPP \\
\hline $\mathbf{4}$ & NSC President \\
\hline $\mathbf{5}$ & Government Presidency \\
\hline $\boldsymbol{\cdots}$ & $\cdots$ \\
\hline
\end{tabular}

Table 2: Network connections.

\begin{tabular}{|lll|}
\hline Source & Target & Label \\
\hline $\mathbf{1 6}$ & 323 & Fax \\
\hline $\mathbf{1 6}$ & 325 & Fax \\
\hline $\mathbf{1 6}$ & 326 & Fax \\
\hline $\mathbf{3 2 5}$ & 368 & Fax \\
\hline $\mathbf{1}$ & 2 & Landline Phone \\
\hline $\mathbf{1}$ & 3 & Landline Phone \\
\hline $\mathbf{. .}$ & $\ldots$ & $\ldots$ \\
\hline
\end{tabular}


The nodes represent the users involved in the NEP and the links the relations between them. Each type of link represents a communication system. In our case study, there are 832 nodes and 12 layers (fax, Internet, landline phone, mobile, satellite, Reman radio channel, Remer radio channel, Civil Guard radio-phone, Radiological Group radio-phone, Autonomous Police radio-phone, in-situ communications, and Beeper). We store the network model in two tables: Table 1 contains all the nodes. Each node, represented as a row, has an id and a label. Table 2 contains all the relations between nodes. A relation, represented as a row, is defined using Source node, Target node and Label (i.e. type of link that handles the relation).

\subsection{Agent Based Model (ABM)}

The Agent-Based model represents the behavior of the users involved in the plan (modeled as agents), the devices (modeled as objects) and the networks that connect the devices (modeled as objects). To define the behavior of the agents, we instantiate the XML file in figure 2 with the specific information of the NEP.

We model the behavior of the devices (e.g. phone, radio, etc.) and networks as objects. For each device, we identify the set of possible states and their behavior in each state. The device may also have different parameters such as the probability of being in a certain state and the delay introduced in the communication. For instance, for the Mobile model, shown in figure 6. We have two parameters: ring duration and delay, and one attribute (id). The Mobile can be in seven states: idle, incoming call requested, outcoming call requested, communication established, transmitting the command, communication over and out of order.

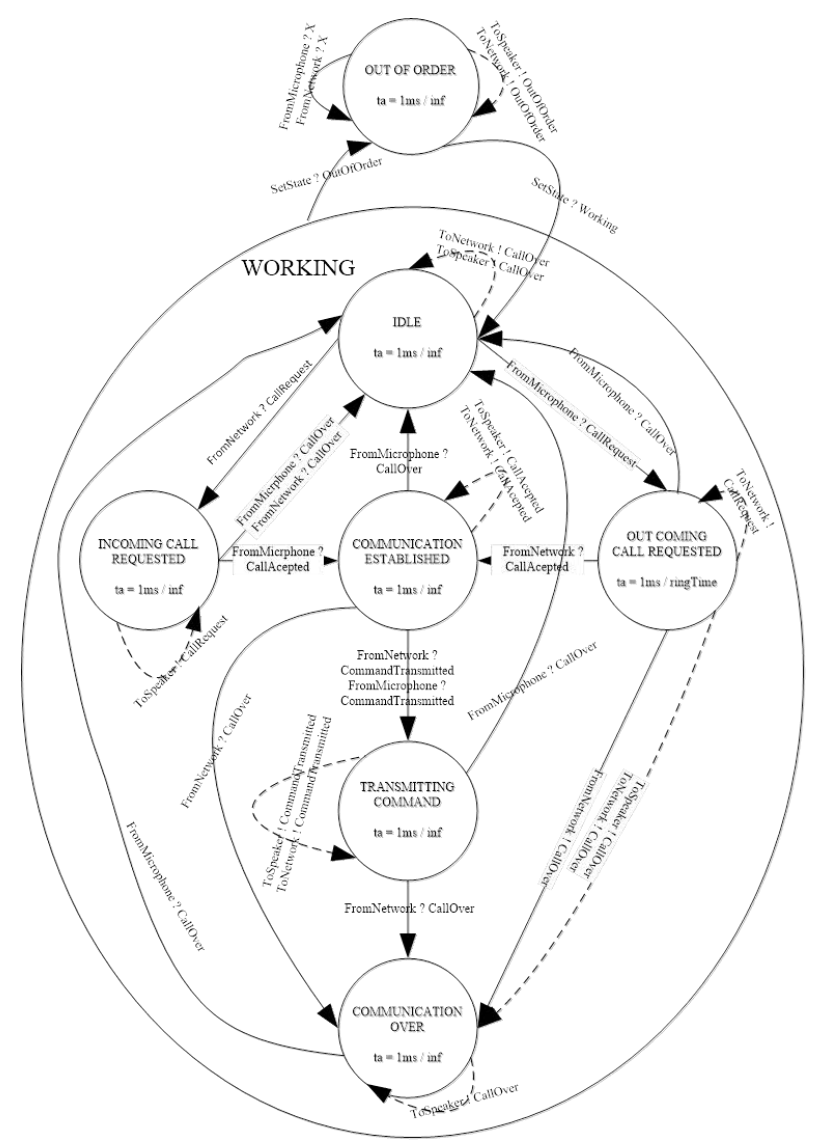

Figure 6: Diagram of the Mobile states using DEVS-Diagram notation.

\subsection{Diffusion Abstract Model (ABM) and Diffusion Computerized Model (DCM)}

We use the Network and the ABM to instantiate the DAM following the architecture presented in section 3. If we need to add additional devices or modify the structure of the XML, we need to define and implement DEVS models of those devices and adapt the program to parse the new XML format; otherwise, we just need to parse the XML and generate the DCM. 


\subsection{Simulation Results}

Once the DAM is implemented (i.e. we have the DCM), we can run different simulations to study the effect of different failures in the communications mechanisms in the emergency plans. Here we show the results of simulating failures in 3 different devices/communication mechanisms (i.e. Mobile phone, Internet/Email, and Landline phone) used during an emergency using a network graph. We choose these communication mechanisms because they are the most widely used during the emergency. We also show how other graphs to identify the users and the devices with more workload can be generated.

The mobile phone is owned by most of the persons in the system and it has one of the highest priorities to be used for both sending and receiving messages. Figure 7 (a) shows the graph generated for the simulation results for the whole network of 832 nodes with a break probability of $20 \%$. We show all the nodes in the system and the active communicating links (i.e. the links among the users in this scenario). The links in the figure represent the communications that have been requested. The colors of the links represent the percentage of successful communications. As we can see, the communication is strong in the core of the network, i.e. all the nodes in the head of the plan receive the messages. Additionally, the first responders also receive the communications successfully. The users with more workload are represented with a bigger size on the network model. In Figure 7 (b), we focus on those nodes that participated in the communication process. Based on the color of the links (light grey), we can conclude that the communications were successful. However, we can see three links in red around node 1. It means that for those communication links, less than $50 \%$ of the messages were transmitted successfully, which means, they need retransmission.

Graph network NEP : Everyone but only communicating links

Graph network NEP : Only people who communicated
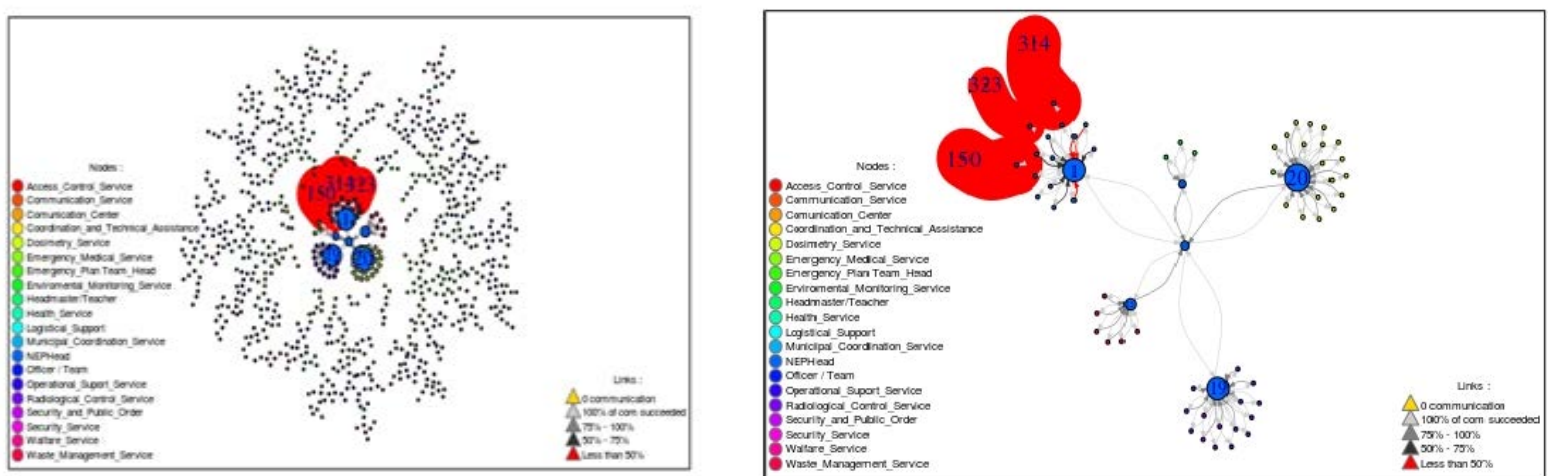

Figure 7: (a) Graph network for mobile at $\mathrm{p}=0.2$ (b) Only communicating links for mobile at $\mathrm{p}=0.2$.

Graph network NEP : Only people who communicated

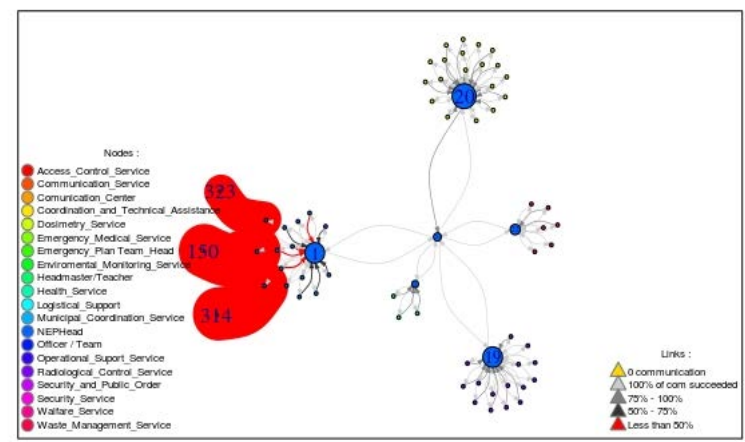

Figure 8: Only communicating for mobile at $\mathrm{p}=0.8$.

Based on these results, we can conclude that for a low probability of $20 \%$, most communications seem to be working and all the nodes are receiving the messages. Therefore, the emergency plan is resilient against a $20 \%$ failure probability in the mobile phone device. If we increase the failure probability to $80 \%$, we can 
see (figure 8 ) that the results are similar to the $20 \%$ failure probability. Therefore, we can conclude that increasing the failure ratio does not affect communications.

E-mail is not used by all the users involved in the emergency, but it is still one of the top priority communication mechanisms. In order to be able to compare the results with the analysis for the mobile phone, we simulate a $20 \%$ and an $80 \%$ failure rate when the same set of messages as in the previous scenario are transmitted. In figures 9 (a) and 9 (b) we show the simulation results for a $20 \%$ failure probability and in figure 10 for an $80 \%$ failure probability. As we can see in the figures, the nodes with more workload remain the same because the same set of messages are transmitted.

Graph network NEP : Everyone but only communicating links

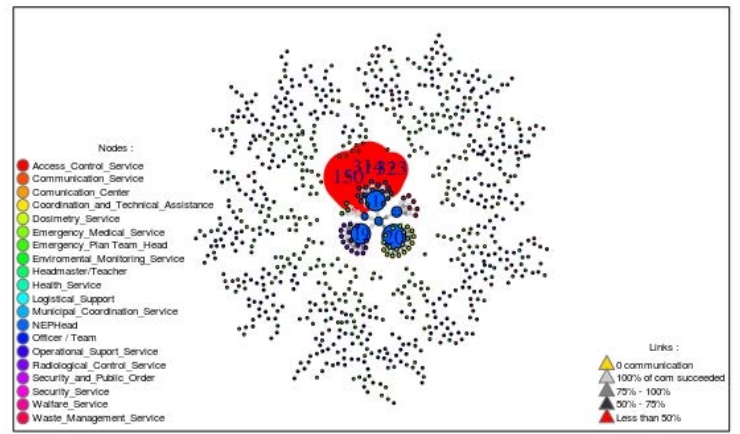

Figure 9: (a) Graph network for email at $\mathrm{p}=0.2$
Graph network NEP : Only people who communicated

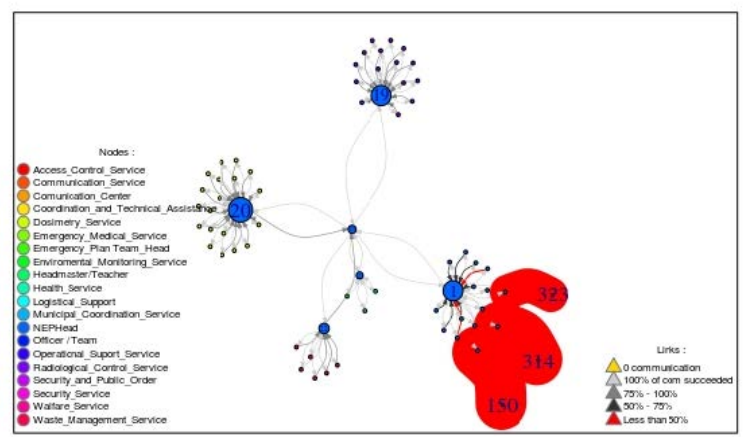

(b) only communicating links for email at $\mathrm{p}=0.2$.

If we focus on how the communications work, when we compare figures 9 and 10, we can see that moving from a $20 \%$ failure rate to an $80 \%$ one has an important impact on the e-mail communications. In figure 9 ( $20 \%$ failure rate), we can see that we have two links in red (less than $50 \%$ of the communications were successful in the first attempt). However, in figure 10 (80\% failure rate), we have the double (i.e. 4 red links). Based on this analysis, we can conclude that the failure rate for email communications is important in terms of resilience.

Graph network NEP : Only people who communicated

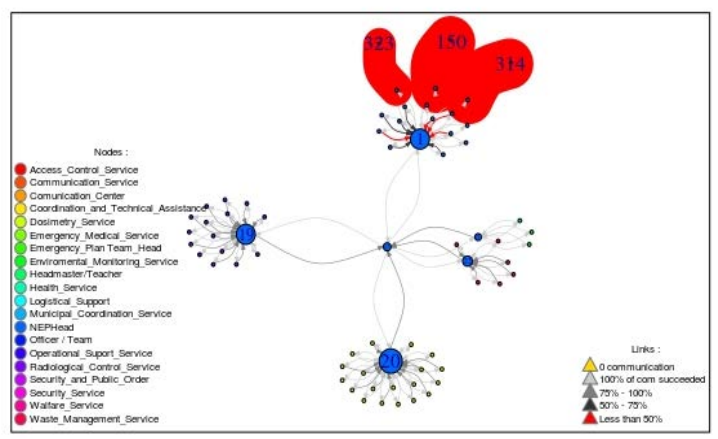

Figure 10: only communicating links for email at $\mathrm{p}=0.8$.

The landline phone is also widely used over the emergency plan. It has higher priority over email but lower priority than the mobile phone. It has a lower priority than the mobile phone because first responders usually do not have access to this communication mechanism. Figure 11 shows the nodes involved in the communication process for failure rates of $20 \%, 50 \%$, and $80 \%$ respectively. In this analysis, we can identify some unexpected results: when the failure rate is low, there are more red links (i.e. less than 50\% of the communications were successful in the first attempt) than when the failure rate is high.

This unexpected emergent behavior is the result of the individual behavior of the users. The behavior defined for the users is as follows: if my preferred communication device is working, I try to communicate using it. If it is not available, I use an alternative communication device if I have one available. When the 
failure probability is low, it is highly likely that my device is working. However, the device used by the person to be contacted may be broken. Therefore, there will be several attempts. However, when the failure probability is high, it is more likely that a device is broken, therefore, we use a different mechanism. These results show the importance and the effect of the behavior of users in the resilience of the emergency plan. These results suggest, the maximum number of attempts a person makes before assuming a communication link is broken is a critical parameter for the resilience of the system.

Graph network NEP : Only people who communicated

Graph network NEP : Only people who communicated
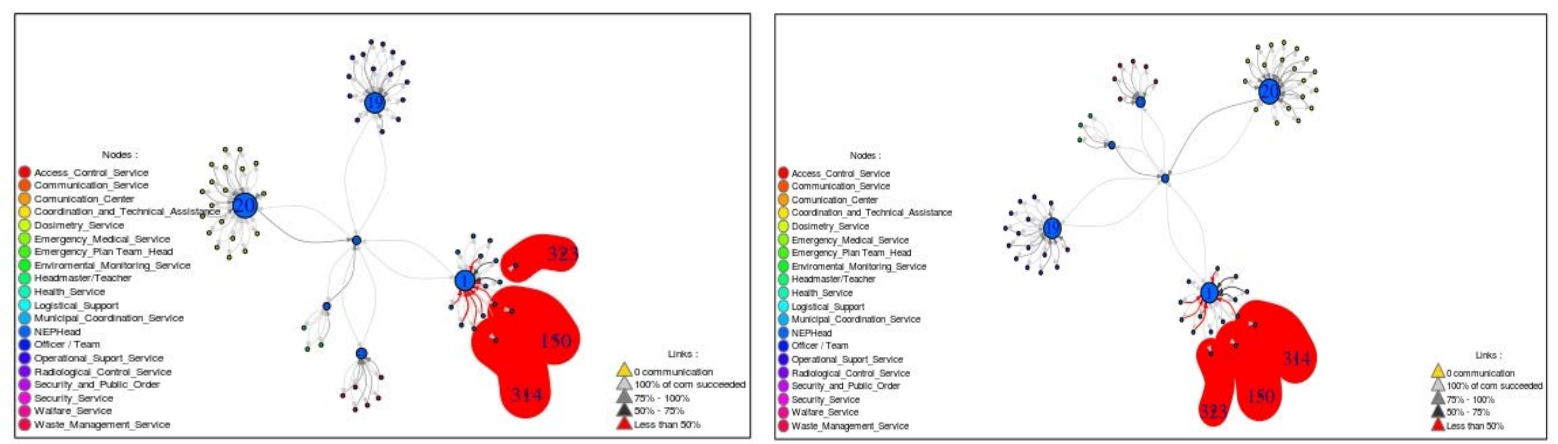

Graph network NEP : Only people who communicated

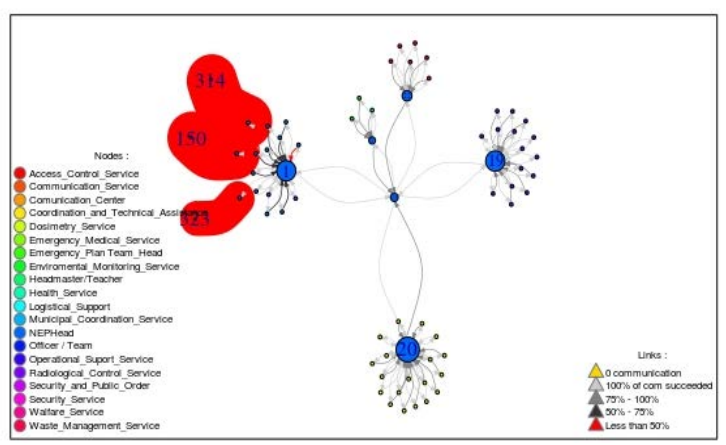

Figure 11: (a) only communicating links for landline at $\mathrm{p}=0.2$, (b) only communicating links for landline at $\mathrm{p}=0.5$, (c) only communicating links for landline at $\mathrm{p}=0.8$.

We can also analyze who are the users with more communication load and the devices that handle more communication requests as shown in figure 12.
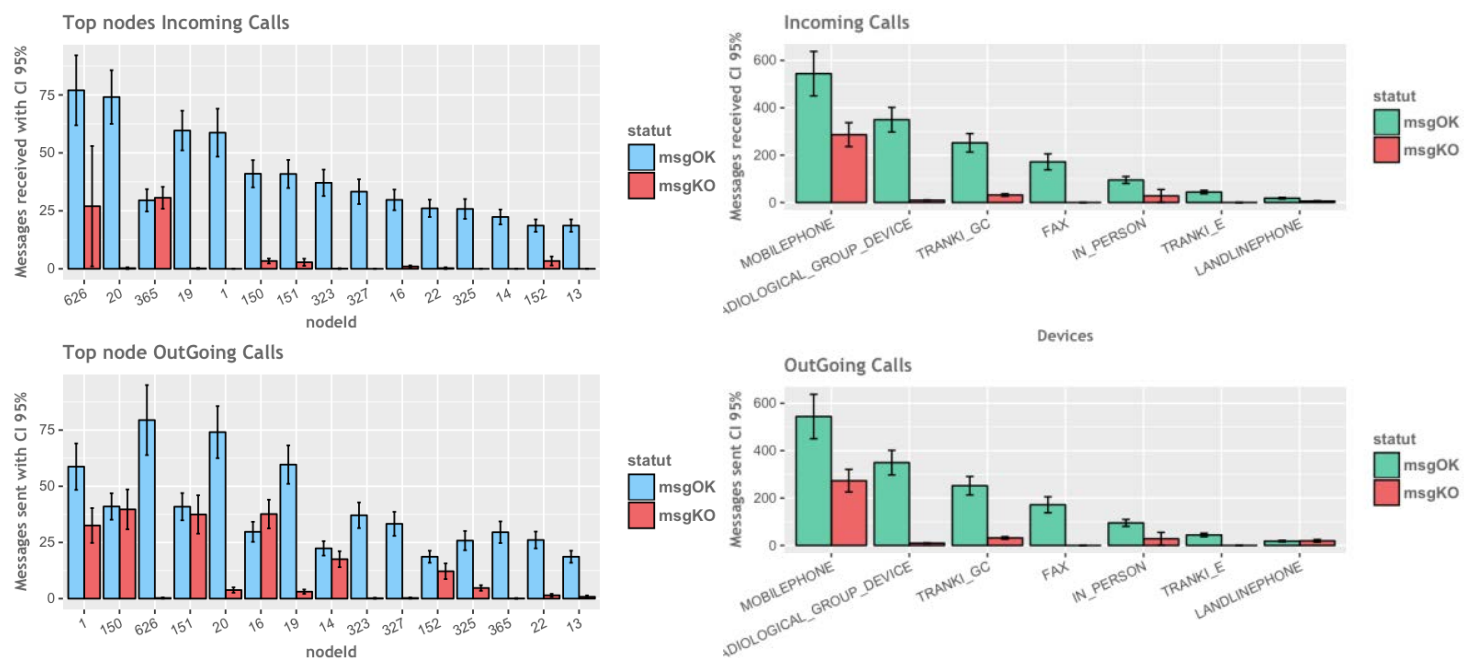

Figure 12: (a) Nodes with more communication classified based on messages. (b) Devices with more communication workload classified based on sent and received messages. 
In figure 12 (a), we analyze the users that receive and send more communication requests. We classify those communication request based on if they were successful communication or not. From this analysis, we can identify if the extra workload of the users is due to the malfunction of the communications or not. In the case of incoming communications, we can see that most of them were successful. However, the results point out that the communications mechanisms for persons with id 626 and 365 should be revised as many of their incoming communications ended ineffectively. The analysis of the outcoming communications reveals that if the communication network or devices have a lower failure rate, the workload of the users would be reduced as they can transmit their message in a single attempt. Based on the same reasoning and the results presented in figure 12 (b), we can reduce the load of the different communication devices reducing their failure rate. As we can see in the figure, many of the communication handled by the devices fail and the users needed to establish a new communication to transmit the message.

\section{CONCLUSIONS}

The framework and a development process presented here is based on a formal modeling and simulation methodology (DEVS), combined with Agent-Based Modeling to represent users and AI Systems and their behavior, and Network Theory to define the relations between these users and AI Systems. We integrate this behavior into the Network to develop the DAM following the architecture presented in section 3 .

The proposed framework and its implementation have the advantages of the general architecture to simulate diffusion processes in multiplex networks proposed in (Ruiz-Martin, Wainer, and Lopez-Paredes, 2018a):

- If the behavior of any agent or any relation (defined in our XML file) changes, we do not need to modify our model or change the implementation. We just update the XML file and run the program that generates the DCM again with the new parameters. This facilitates running different scenarios where the connections between agents and/or the agent behavior vary. It also allows us to run a subset of the emergency plan such as a specific group just reducing the XML files that the program takes as input to this subset of users.

- It reduces implementation time as the top model is automatically built based on the XML where the behavior of the agents is defined.

- It facilitates model reusability. For example, if we want to update a model of the architecture (e.g., we want to replace the Mobile phone atomic model for a more accurate one), we just need to define the new atomics inside the coupled model. The rest of the models are still useful. Therefore, this implementation saves time and effort in the model definition, implementation, and verification.

Analyzing the simulation results we obtain applying our architecture, we can propose improvements to the emergency plan. For example, as we showed in the previous section, if we identify the communication links that are more prone to fail, we can define alternative communication mechanisms.

\section{REFERENCES}

Bouanan, Y. 2016. "Contribution à une architecture de modélisation et de simulation à événements discrets: application à la propagation d'information dans les réseaux sociaux" $\mathrm{PhD}$ Thesis, Univ. of Bordeaux.

Chewning, L., C Lai, and M Doerfel. 2012. "Organizational Resilience and Using Information and Communication Technologies to Rebuild Communication Structures.” Mgmt Comm Q 27 (2): 237-63.

De Maio, C., G. Fenza, M. Gaeta, V. Loia, and F. Orciuoli. 2011. "A Knowledge-Based Framework for Emergency DSS.” Knowledge-Based Systems 24: 1372-79.

Espinosa-Paredes, G., A Nuñez-Carrera, A Laureano-Cruces, A Vázquez-Rodríguez, and E EspinosaMartinez. 2008. "Emergency Management for a Nuclear Power Plant Using Fuzzy Cognitive Maps." Annals of Nuclear Energy 35 (12): 2387-96.

European Commission. 2017. "Resilience as Strategic Priority of the External Action of the EU. RoadmapAres 1137007."https://ec.europa.eu/info/law/better-regulation/initiatives/ares-2017-1137007_en. 
Garnett, J., and A. Kouzmin. 2007. "Communicating throughout Katrina: Competing and Complementary Conceptual Lenses on Crisis Communication.” Public Administration Review 67: 171-88.

Government of Canada. 2014. Canada in a Changing Climate: Sector Perspectives on Impacts and Adaptation. Edited by F.J. Warren and D.S. Lemmen. Ottawa, ON.

Kachali, H., J. R. Stevenson, Z. Whitman, E. Seville, J. Vargo, and T. Wilson. 2012. "Organisational Resilience and Recovery for Canterbury Organisations after the 4 September 2010 Earthquake." Australasian Journal of Disaster and Trauma Studies 1 (1):11-19.

Kendra, J., and T. Wachtendorf. 2003. "Elements of Resilience after the World Trade Center Disaster: Reconstituting New York City’s Emergency Operations Centre.” Disasters 27 (1): 37-53.

Langlois, L. 2013. "IAEA Action Plan on Nuclear Safety.” Energy Strategy Reviews 1 (4): 302-306.

Longstaff, PH, and S. Yang. 2008. "Communication Management and Trust: Role in Building Resilience to 'Surprises' Such as Natural Disasters, Pandemic Flu, and Terrorism.” Ecology and Society 13(1):3.

MacKenzie, C., J. Santos, and K. Barker. 2012. "Measuring Changes in International Production from Disruption: Case Study of Japanese Tsunami." Intl J of Production Economics138 (2): 293-302.

Mendonça, D., G. Beroggi, D. van Gent, and W. Wallace. 2006. "Designing Gaming Simulations for the Assessment of Decision Support Systems in Emergency Response." Safety Science 44(6):523-35.

Mendonça, D., and W. Wallace. 2015. "Factors Underlying Organizational Resilience: Electric Power Restoration in New York City after 11 Sep 2001." Reliability Eng \& System Safety 141(0):83-91.

Omoto, A. 2013. "The Accident at TEPCO's Fukushima-Daiichi Nuclear Power Station: What Went Wrong and What Lessons Are Universal?" Nuclear Instruments and Methods in Physics Research Section A: Accelerators, Spectrometers, Detectors and Associated Equipment 731: 3-7.

Ruiz-Martin, C. 2013. "Modelo Organizacional para la Gestión de Emergencias" Universidad de Valladolid. Master Thesis.

Ruiz-Martin C., Y.Bouanan, G. Wainer, G. Zacharewicz, and A. Lopez-Paredes. 2016 “A Hybrid Approach to Study Communication in Emergency Plans". Winter Simulation Conference, Arlington, VA.

Ruiz-Martin, C. 2017. "An Architecture to Simulate Diffusion Processes in Multiplex Dynamic Networks." In Proceedings of the 2017 Winter Simulation Conference (WSC), Las Vegas, USA, 4630-31. IEEE.

Ruiz-Martin, C., G. Wainer, and A. Lopez-Paredes. 2018a. "Formal Abstract Modeling of Dynamic Multiplex Networks." ACM Conference on Principles of Advanced Discrete Simulation. Rome, Italy.

Ruiz-Martin, C., A. López-Paredes, and G. Wainer. 2018b. "What we Know and do not Know about Organizational Resilience". International J. of Production Management and Engineering, 6(1): 11-28.

Vicino, D., L. Belolli, C. Ruiz-Martin, G. Wainer. "Building DEVS Models with the Cadmium Tool". Proceedings of Winter Simulation Conference. National Harbor. MD. 2019.

Whitman, Z., J. Stevenson, H. Kachali, E. Seville, J. Vargo, and T. Wilson. 2014. "Organisational Resilience Following the Darfield Earthquake of 2010." Disasters 38 (1): 148-77.

Zeigler, B.P., H. Praehofer, and T. G. Kim, 2000. Theory of modeling and simulation. Academic press.

\section{AUTHOR BIOGRAPHIES}

CRISTINA RUIZ-MARTIN is a Postdoctoral Fellow at the Department of Systems and Computer Engineering at Carleton University. Her email address is cristinaruizmartin@sce.carleton.ca.

GABRIEL WAINER is a Full Professor at the Department of Systems and Computer Engineering at Carleton University. He is a Fellow of SCS. His email address is gwainer@sce.carleton.ca

ADOLFO LOPEZ-PAREDES is a Full Professor at the Department of Business Management at the Industrial Engineering School at UVa. His email address is aparedes@eii.uva.es. 